\title{
Dismantlability, Connectedness, and Mixing in Relational Structures
}

Raimundo Briceño

School of Mathematical Sciences, Tel Aviv University, Tel Aviv 69978, Israel

raimundo@alumni.ubc.ca

Andrei A. Bulatov

School of Computing Science, Simon Fraser University, Canada

abulatov@sfu.ca

Víctor Dalmau

Department of Information and Communication Technologies, Universitat Pompeu Fabra, Barcelona, Spain

victor.dalmau@upf.edu

\section{Benoît Larose}

LACIM, Université du Québec a Montréal, Montréal, Canada

blarose@lacim.ca

\begin{abstract}
The Constraint Satisfaction Problem (CSP) and its counting counterpart appears under different guises in many areas of mathematics, computer science, statistical physics, and elsewhere. Its structural and algorithmic properties have demonstrated to play a crucial role in many of those applications. For instance, topological properties of the solution set such as connectedness is related to the hardness of CSPs over random structures. In approximate counting and statistical physics, where CSPs emerge in the form of spin systems, mixing properties and the uniqueness of Gibbs measures have been heavily exploited for approximating partition functions or the free energy of spin systems. Additionally, in the decision CSPs, structural properties of the relational structures involved - like, for example, dismantlability - and their logical characterizations have been instrumental for determining the complexity and other properties of the problem.

In spite of the great diversity of those features, there are some eerie similarities between them. These were observed and made more precise in the case of graph homomorphisms by Brightwell and Winkler, who showed that the structural property of dismantlability of the target graph, the connectedness of the set of homomorphisms, good mixing properties of the corresponding spin system, and the uniqueness of Gibbs measure are all equivalent. In this paper we go a step further and demonstrate similar connections for arbitrary CSPs. This requires much deeper understanding of dismantling and the structure of the solution space in the case of relational structures, and new refined concepts of mixing introduced by Briceño. In addition, we develop properties related to the study of valid extensions of a given partially defined homomorphism, an approach that turns out to be novel even in the graph case. We also add to the mix the combinatorial property of finite duality and its logic counterpart, FO-definability, studied by Larose, Loten, and Tardif.
\end{abstract}

2012 ACM Subject Classification Mathematics of computing $\rightarrow$ Paths and connectivity problems

Keywords and phrases relational structure, constraint satisfaction problem, homomorphism, mixing properties, Gibbs measure

Digital Object Identifier 10.4230/LIPIcs.ICALP.2019.29

Category Track A: Algorithms, Complexity and Games

Related Version A full version of this paper is available at http://arxiv.org/abs/1901.04398.

Funding This work was done in part while the first three authors were visiting the Simons Institute for the Theory of Computing at University of California, Berkeley.

Raimundo Briceño: The first author was supported by ERC Starting Grants 678520 and 676970.

(c) (i) Raimundo Briceño, Andrei A. Bulatov, Víctor Dalmau, and Benoît Larose;

cc) licensed under Creative Commons License CC-BY

46th International Colloquium on Automata, Languages, and Programming (ICALP 2019). Editors: Christel Baier, Ioannis Chatzigiannakis, Paola Flocchini, and Stefano Leonardi;

Article No. 29; pp. 29:1-29:15

Leibniz International Proceedings in Informatics

LIPICS Schloss Dagstuhl - Leibniz-Zentrum für Informatik, Dagstuhl Publishing, Germany 
Andrei A. Bulatov: This work was supported by an NSERC Discovery grant. Victor Dalmau: The third author was supported by MICCIN grant TIN2016-76573-C2-1P and Maria de Maeztu Units of Excellence Programme MDM-2015-0502.

Benoit Larose: The fourth author was supported by an NSERC Discovery grant and FRQNT.

\section{Introduction}

The Constraint Satisfaction Problem (CSP) provides a powerful framework in a wide range of areas of mathematics, computer science, statistical physics, and elsewhere. The goal in a CSP is to find an assignment to variables from a given set that satisfies a number of given constraints. The counting version of the problem asks about the number of such assignments. The CSP however appears in different forms: as the standard one outlined above in AI and computer science [19], as the homomorphism problem in graph and model theory [22, 27], as conjunctive query evaluation in logic and database theory [30], as computing the partition function of a spin system in statistical physics [39] and related areas, like symbolic dynamics and coding $[35,37]$.

The CSP allows for many approaches of diverse nature, and every application field exploits some of its many facets: structural properties of constraints for complexity and algorithms, probabilistic properties and the topology of the solution space in Random CSP and random structures, mixing properties in statistical physics and dynamical systems, decay of correlations and the uniqueness of probabilistic measures in approximate counting, and homomorphic duality and logical characterizations in model theory. In [12], Brightwell and Winkler observed that some of these properties are actually closely related, at least in the simple case of graph homomorphisms. In this paper we take this research direction a step further by extending Brightwell and Winkler's results to the general CSP, and by refining and widening the range of the properties involved.

We start off with a brief introduction of the features of the CSP considered in this paper. Afterwards, we provide a detailed account of the necessary background and a description of our results.

Every CSP involves a set of variables and a domain, a set of possible values for the variables. Assumptions about these two sets differ in different areas. The most studied case in combinatorics and complexity theory is when both sets are finite. However, many interesting problems such as scheduling and temporal and spatial reasoning involve infinite domains; see also extensive literature on infinite CSPs (see, for example, [4] and the references therein). In other cases such as in statistical physics, it is natural to choose the set of variables to be infinite (a lattice, for example). Then, it is also natural to study probability distributions over such assignments - where Gibbs measures and the problem of their (non-) uniqueness appear naturally [25] - and also study quantities such as entropy and free energy [3, 7].

Following [22], CSPs can be formulated as the problem of deciding the existence of a homomorphism from a finite relational structure $\mathbb{G}$ to a target relational structure $\mathbb{H}$, where $\mathbb{G}$ and $\mathbb{H}$ encode the variables and the values of the CSP. The complexity of this problem, especially the case when $\mathbb{H}$ is a fixed finite relational structure, has received a lot of attention, culminating with the proof of the Feder-Vardi conjecture [14, 44]. In the present paper we focus as well on the case when $\mathbb{H}$ is finite, although our main focus is not algorithmic but rather structural. In particular, we are interested in studying the space $\operatorname{Hom}(\mathbb{G}, \mathbb{H})$ of homomorphism from $\mathbb{G}$ to $\mathbb{H}$. Furthermore, following [12] we consider homomorphisms from both finite and infinite relational structures $\mathbb{G}$ (although [12] only considers graphs), a flexibility that turns out to be useful to see different aspects of homomorphism spaces $\operatorname{Hom}(\mathbb{G}, \mathbb{H})$ that otherwise would be meaningless. 
There is a vast literature concerning graph homomorphisms and their properties through the lenses of statistical physics $[5,20,11,39]$. In this context, it is very common to encode a spin system as a pair of relational structures $\mathbb{G}$ and $\mathbb{H}$, where $\mathbb{G}$ contains a set of variables/ particles and $\mathbb{H}$ contains the set of values/spins that each particle could take, imposing hard constraints on them, i.e., disregarding configurations of values that do not satisfy all the given constraints. In practical terms, all this reduces to study - individually and as a set the maps from $\mathbb{G}$ to $\mathbb{H}$ that are homomorphisms. In particular, many important parameters of a spin system such as free energy and entropy can be learned from studying such a set of homomorphisms.

In [12], Brightwell and Winkler observed that many of the properties of graph homorphisms used in the above areas are equivalent to a single structural property of graphs, namely, dismantlability. In this paper we follow a similar approach and study properties of CSPs over general relational structures that we put into basically three categories: (1) dismantlability, (2) connectedness, and (3) mixing. Furthermore, as a consequence of our results, we established a connection with a fourth notion not initially contemplated in [12]: (4) finite duality.

\section{Dismantlability}

A graph is said to be dismantlable if it can be reduced to a single vertex removing vertices whose neighborhood is contained in the neighborhood of some other vertex. Such transformations are called folds, and they can be viewed as retractions of a very particular kind. Dismantlable graphs were introduced in [41], based on ideas already present in [29] in the context of lattices, and have been intensively studied in combinatorics. Distamantlability can be generalized in a natural way to relational structures. Indeed, some variants of this notion have been used in the study of CSPs. In particular, dismantlability has been applied in [15] to the problem of enumerating all solutions of $\operatorname{Hom}(\mathbb{G}, \mathbb{H})$ with polynomial delay. Also, it has played a major role in the study of CSPs definable in first-order logic [18, 34].

\section{Connectedness}

When $\mathbb{G}$ is finite, it is often useful to convert $\operatorname{Hom}(\mathbb{G}, \mathbb{H})$ into a graph and explore the connectivity properties of this graph. The set of edges of $\operatorname{Hom}(\mathbb{G}, \mathbb{H})$ can be defined in a variety of ways, usually the most suitable to the problem at hand. For example, it is common to say that two elements from $\operatorname{Hom}(\mathbb{G}, \mathbb{H})$ are close (and therefore adjacent in the graph) if the Hamming distance between them is smaller than a certain threshold. The particular case when this threshold is 1 has been intensively studied, motivated initially by the fact that the connectedness of the solution space for SAT problems over random instances is linked to the performance of standard satisfiability algorithms, such as WalkSAT or DPLL $[1,32]$. This has given rise to a general framework called reconfiguration [28] (see also [40] for a recent survey) that goes way beyond homomorphisms. Work in this area encompasses both structural questions (under which conditions is $\operatorname{Hom}(\mathbb{G}, \mathbb{H})$ connected?) and algorithmic ones (what is the complexity of, deciding, given $\mathbb{G}$ and $\mathbb{H}$ as input, whether $\operatorname{Hom}(\mathbb{G}, \mathbb{H})$ is connected? Its diameter? The shortest path between two given members of $\operatorname{Hom}(\mathbb{G}, \mathbb{H})$ ? Etc.). In the context of spin systems, the connectedness of $\operatorname{Hom}(\mathbb{G}, \mathbb{H})$ is related to processes that consists on periodically updating the spin of a single or a small set of particles (e.g., irreducibility of Glauber dynamics).

We also consider an alternative way to define adjacency in $\operatorname{Hom}(\mathbb{G}, \mathbb{H})$ via links as in [34]. This notion of adjacency is linked to the so-called finite duality property, which is another of the main themes of our work. 


\section{Mixing}

Mixing properties have been intensively studied in statistical physics and related areas (see $[2,6,8,10,17,42]$ ), and are usually applied when the set of particles in $\mathbb{G}$ is very large or infinite. In this case, it can be very useful to be able to "glue" together partial homomorphisms, provided their domains are far from each other. There are several properties that formalize this phenomenon and it is common to establish hierarchies among them. More concretely, given a metric in $\mathbb{G}$, it is natural to ask whether there exists some uniform gap such that for any two subsets $A$ and $B$ of particles sufficiently far apart (in terms of the gap), and for any pair of homomorphisms $\phi, \psi \in \operatorname{Hom}(\mathbb{G}, \mathbb{H})$, we can find a third one, $\gamma$, such that restriction of $\gamma$ to $A$ and $B$ coincides with the restrictions of $\phi$ and $\psi$ on $A$ and $B$, respectively. On the contrary, whenever the information content of a given set (at least partially) determines the information content of another set (i.e., the possible values that the variables on it can take), no matter how far it is, such a phenomenon has been called long range action in previous work (e.g., see [13]).

Similar phenomena are used in the related area of approximate computing of partition functions, where many algorithms are based on decay of correlations between values of remote elements of $\mathbb{G}$, which allows for approximation of partition functions based only on local neighborhoods of variables.

\section{Finite duality and logic characterizations}

Homomorphism duality often helps to design a solution algorithm for a CSP or establish its useful properties. A graph (or relational structure) $\mathbb{H}$ is said to have homomorphism duality if there is a set $\mathcal{O}$ of graphs, called obstructions, such that a graph $\mathbb{G}$ has a homomorphism to $\mathbb{H}$ if and only if no graph from $\mathcal{O}$ is homomorphic to $\mathbb{G}$. Sometimes the set of obstructions is very simple, say, any bipartite graph has homomorphic duality, where $\mathcal{O}$ is the set of all odd cycles. If $\mathcal{O}$ can be chosen finite, we say that $\mathbb{H}$ has finite duality.

Homomorphism duality is closely related to another property of CSPs. Let $\mathcal{L}$ be a logic language such as first order, second order, etc. The problem of deciding homomorphisms to a relational structure $\mathbb{H}$ is said to be expressible in $\mathcal{L}$ if there is a formula $\Phi$ in the language $\mathcal{L}$ such that $\mathbb{G}$ has a homomorphism to $\mathbb{H}$ if and only if $\Phi$ is true on $\mathbb{G}$. It is known, for instance, that $\mathbb{H}$ has a set of obstructions consisting of relational structures of bounded treewidth if and only if the corresponding homomorphism problem is expressible in Datalog [22], or that $\mathbb{H}$ has finite duality if and only if the corresponding problem is expressible in first order logic [34] (see, for example, [16] for a survey on dualities for CSP).

\section{Our results}

The main result of this paper is Theorem 10, that shows, for a relational structure $\mathbb{H}$, the equivalence of the following three conditions: (A) $\mathbb{H}^{2}$ dismantles to a substructure of its diagonal, that is, the substructure of $\mathbb{H}^{2}$ induced by the set $\{(a, a) \mid a \in \mathbb{H}\}$; (B) for any $\mathbb{G}$, the homomorphism graph $\operatorname{Hom}(\mathbb{G}, \mathbb{H})$ is connected; and $(\mathrm{C})$ for any relational structure $\mathbb{G}$, the graph $\operatorname{Hom}(\mathbb{G}, \mathbb{H})$ satisfies certain mixing properties. These results generalize the results from [12] to the case of general relational structures. Observe that the case of graphs considered in [12] does not fully reflect the richness of the theory behind our result.

As a byproduct of our results, we obtain two applications. On the one hand, we establish a link with strong spatial mixing (e.g., see [21]) and topological strong spatial mixing (introduced in [7]). These two last properties have played an important role in the development of deterministic approximate counting algorithms. In this paper we address the 
following question: What fixed targets $\mathbb{H}$ are suitable for both of these properties to hold for any $\mathbb{G}$ ? On the other hand, we establish a connection with finite duality, which allows us to reprove the main theorem in [34]. We hope that our work opens the possibility of developing new counting techniques based on this approach in a very general setting. We stress that many of these results are new even in the graph case.

Due to space restrictions proofs are ommitted. They can be found at the full version [9].

\section{Preliminaries}

Let $H$ be a countable (finite or denumerable) set and $k$ a positive integer. The set of $k$-tuples over $H$ is denoted by $H^{k}$. A ( $k$-ary) relation $R$ over $H$ is a subset $R \subseteq H^{k}$. The elements of a relation $R$ will be denoted in boldface, e.g., $\mathbf{a}, \mathbf{b}$, etc., and $\mathbf{a}[i]$ will denote the $i$ th entry of a for $1 \leq i \leq k$.

Given another countable set $G$ and a map $\phi: G \rightarrow H$, for a $k$-tuple a over $G$ we shall use $\phi(\mathbf{a})$ to denote the $k$-tuple over $H$ obtained after applying $\phi$ to a componentwise. If $V \subseteq G$, we will denote by $\left.\phi\right|_{V}$ the restriction of $\phi$ to $V$. Furthermore, if $\psi$ is another map with domain $H$, we shall use $\psi \circ \phi$ to denote the composition of $\psi$ with $\phi$, i.e., the map $x \mapsto \psi(\phi(x))$.

A signature $\tau$ is a finite collection of relation symbols $R$, each of them with an associated arity. For a given signature $\tau$, a relational structure (with signature $\tau$ ) - or simply, a $\tau$-structure $-\mathbb{H}$ consists of a countable set $H$ called the universe of $\mathbb{H}$ and a relation $R(\mathbb{H})$ for each $R \in \tau$, such that the arity of $R(\mathbb{H})$ equals that of $R$. We shall use the same capital letter to denote the universe of a $\tau$-structure, e.g., $H$ is the universe of $\mathbb{H}$. We will usually consider $\tau$ to be a fixed signature, and $\mathbb{G}$ and $\mathbb{H}$ to be $\tau$-structures with universes $G$ and $H$, respectively.

A relational structure is said to be finite if its universe is finite and locally finite if every element in its universe occurs only in a finite number of its tuples.

- Remark 1. A digraph $\mathbb{G}$ (with self-loops allowed) is a very particular case of a relational structure, where the signature $\tau$ consists of a unique relation symbol $E$ of arity 2. Moreover, graphs correspond to the digraph case where $E(\mathbb{G})$ is a symmetric relation.

A map $\phi: G \rightarrow H$ is said to be a homomorphism from $\mathbb{G}$ to $\mathbb{H}$ if, for every relation symbol $R \in \tau$,

$\mathbf{a} \in R(\mathbb{G}) \Rightarrow \phi(\mathbf{a}) \in R(\mathbb{H})$.

We will denote by $\operatorname{Hom}(\mathbb{G}, \mathbb{H})$ the set of all homomorphisms from $\mathbb{G}$ to $\mathbb{H}$.

- Example 2. A particular example of CSPs that cannot be represented in the setting of Brightwell and Winkler (that is, as homomorphisms of graphs) is the case of $d$-dimensional nearest-neighbor (n.n.) shifts of finite type (SFTs), a fundamental object in dynamical systems and probability (see $[35,36,37]$ ). Given a positive integer $d$, consider the signature $\tau=\left\{R_{1}, \ldots, R_{d}\right\}$, where $R_{i}$ is a 2 -ary relation for all $1 \leq i \leq d$. We consider two $\tau$-structures $\mathbb{G}$ and $\mathbb{H}$. Here, $\mathbb{G}$ will be an infinite relational structure with universe $G=\mathbb{Z}^{d}$ and relations $R_{i}(\mathbb{G}), 1 \leq i \leq d$, representing the usual $d$-dimensional hypercubic lattice and the adjacency of pairs of elements in it. On the other hand, $\mathbb{H}$ will be a finite relational structure with universe $H$ and $R_{i}(\mathbb{H})$ representing pairs of "colors" from $H$ that are allowed to be adjacent in the canonical $i$ th direction of the lattice, $1 \leq i \leq d$. Then, $X=\operatorname{Hom}(\mathbb{G}, \mathbb{H})$ is known as a $d$-dimensional n.n. SFT, a set of colorings of $\mathbb{Z}^{d}$ with not necessarily isotropic adjacency rules (i.e., we do not need to have the same restrictions in every direction), and any such object can be represented in this way. 
A relational structure $\mathbb{J}$ is a substructure of $\mathbb{H}$ if $J \subseteq H$ and, for every relation symbol $R \in \tau$, we have that $R(\mathbb{J}) \subseteq R(\mathbb{H})$. Furthermore, if for every $k$-ary $R \in \tau$, we have that $R(\mathbb{J})=R(\mathbb{H}) \cap J^{k}$, then we say that $\mathbb{J}$ is the substructure of $\mathbb{H}$ induced by $J$. If $J \subseteq H$ and $\phi: H \rightarrow J$ is a homomorphism acting as the identity on $J$, then $\phi$ is said to be a retraction.

The product of $\mathbb{H}_{1}$ and $\mathbb{H}_{2}$, denoted $\mathbb{H}_{1} \times \mathbb{H}_{2}$, is the $\tau$-structure with universe $H_{1} \times H_{2}$ where, for every $k$-ary relation symbol $R \in \tau$, we have that $R\left(\mathbb{H}_{1} \times \mathbb{H}_{2}\right)$ consists of all tuples $\left(\left(a_{1}, b_{1}\right), \ldots,\left(a_{k}, b_{k}\right)\right)$ with $\left(a_{1}, \ldots, a_{k}\right) \in R\left(\mathbb{H}_{1}\right)$ and $\left(b_{1}, \ldots, b_{k}\right) \in R\left(\mathbb{H}_{2}\right)$. We shall denote by $\mathbb{H}^{2}$ the product $\mathbb{H} \times \mathbb{H}$. The projections $\pi_{1}, \pi_{2}: H^{2} \rightarrow H$ are the maps $(a, b) \mapsto a$ and $(a, b) \mapsto b$, respectively, for $(a, b) \in H^{2}$. An element $(a, b)$ of $H^{2}$ is diagonal if $a=b$. The diagonal set of $H^{2}$, denoted $\Delta\left(H^{2}\right)$, is the set of its diagonal elements. Similarly, the diagonal structure of $\mathbb{H}^{2}$, denoted $\Delta\left(\mathbb{H}^{2}\right)$, is the substructure of $\mathbb{H}^{2}$ induced by $\Delta\left(H^{2}\right)$. A substructure $\mathbb{K}$ of $\mathbb{H}^{2}$ is symmetric whenever $(a, b) \in K$ if and only if $(b, a) \in K$. Notice that $\mathbb{H}^{2}$ is always symmetric.

In this paper, we will study properties of $\mathbb{H}$ and how they relate to other properties of $\operatorname{Hom}(\mathbb{G}, \mathbb{H})$ for arbitrary $\mathbb{G}$. We mainly consider three families of properties, namely, dismantling of $\mathbb{H}$, connectedness of some particular graphs with vertex set $\operatorname{Hom}(\mathbb{G}, \mathbb{H})$, and mixing properties of $\operatorname{Hom}(\mathbb{G}, \mathbb{H})$.

\subsection{Dismantling}

Let $\mathbb{H}$ be a $\tau$-structure and let $a, b$ be elements in its universe $H$. We say that $b$ dominates $a$ (in $\mathbb{H})$ if for every $k$-ary $R \in \tau$, any $i \in\{1, \ldots, k\}$, and any $\left(a_{1}, \ldots, a_{k}\right) \in R(\mathbb{H})$ with $a_{i}=a$, we also have that $\left(a_{1}, \ldots, a_{i-1}, b, a_{i+1}, \ldots, a_{k}\right) \in R(\mathbb{H})$. Additionally, if $a \neq b$, then we say that $a$ is dominated (in $\mathbb{H}$ ).

A sequence of $\tau$-structures $\mathbb{J}_{0}, \ldots, \mathbb{J}_{\ell}$ is a dismantling sequence if for every $0 \leq j<\ell$ there exist $a_{j}, b_{j} \in J_{j}$ such that $b_{j}$ dominates $a_{j}$ in $\mathbb{J}_{j}$, and $\mathbb{J}_{j+1}$ is the substructure of $\mathbb{J}_{j}$ induced by $J_{j} \backslash\left\{a_{j}\right\}$. In this case, we say that $\mathbb{J}_{0}$ dismantles to $\mathbb{J}_{\ell}$. We can alternatively denote a dismantling sequence by giving the initial relational structure $\mathbb{J}_{0}$ and the sequence of elements $a_{0}, \ldots, a_{\ell-1}$. We say that $\mathbb{H}$ is dismantlable if it dismantles to a $\tau$-structure such that its universe is a singleton.

Note that for every $0 \leq j<\ell$ there is a natural retraction $r_{j}$ from $\mathbb{J}_{j}$ to $\mathbb{J}_{j+1}$, where $r_{j}$ maps $a_{j}$ to $b_{j}$ and acts as the identity elsewhere. We call such retractions a fold. By successive composition, one can define a retraction (namely, $r_{j^{\prime}-1} \circ \cdots \circ r_{j}$ ) from $\mathbb{J}_{j}$ to $\mathbb{J}_{j^{\prime}}$ for every $j \leq j^{\prime}$.

It is well known that if $\mathbb{H}$ dismantles to some substructure $\mathbb{K}$, then this dismantling can be found in a greedy manner. Formally,

- Lemma 3 ([34, Lemma 5.1]). If $\mathbb{H}$ dismantles to $\mathbb{K}$ and $a \in H \backslash K$ is dominated in $\mathbb{H}$, then the substructure of $\mathbb{H}$ induced by $H \backslash\{a\}$ dismantles to $\mathbb{K}$.

Let $J \subseteq H$. We say that $\mathbb{H}$ is $J$-non-foldable if every dominated element in $\mathbb{H}$ belongs to $J$.

\subsection{Walks in relational structures}

We define a walk $w$ in a $\tau$-structure $\mathbb{H}$ to be a sequence

$$
a_{0}, i_{1},\left(R_{1}, \mathbf{a}_{1}\right), j_{1}, a_{1}, \ldots, a_{n-1}, i_{n},\left(R_{n}, \mathbf{a}_{n}\right), j_{n}, a_{n}
$$

for some $n \geq 0$, such that, for all $1 \leq \ell \leq n$,

- $R_{\ell} \in \tau, \mathbf{a}_{\ell} \in R_{\ell}(\mathbb{H}), i_{\ell} \neq j_{\ell}$, and

- $a_{\ell-1}=\mathbf{a}_{\ell}\left[i_{\ell}\right]$ and $a_{\ell}=\mathbf{a}_{\ell}\left[j_{\ell}\right]$. 
In this case, we will say that $w$ joins $a_{0}$ (the starting point) and $a_{n}$ (the ending point), and that the length of the walk $w$ is $n$. Notice that if a walk $w$ joins $a_{0}$ and $a_{n}$, then there is another walk $w^{\prime}$ that joins $a_{n}$ and $a_{0}$ obtained by just reversing the order of indices. The distance $\operatorname{dist}(a, b)$ between two elements $a, b \in H$ is defined to be the smallest length among all the walks $w$ that join $a$ and $b$. The distance $\operatorname{dist}(V, W)$ between sets $V, W \subseteq H$ is defined to be the minimum distance between an element from $V$ and an element from $W$.

Note that the definition of walk above coincides with the standard definition of walk when $\mathbb{H}$ is a graph. However, in the case of graphs it will be convenient to describe the walk merely as the list $a_{0}, \ldots, a_{n}$ of its nodes, as usual.

A $\tau$-structure $\mathbb{H}$ is connected if there is a walk joining any pair of elements of its universe $H$ and a connected component is any induced substructure that is connected and maximal in the sense of inclusion. A walk $w$ is a circuit if $n>0$, the starting and ending points of $w$ coincide, and for all $1 \leq \ell<\ell^{\prime} \leq n$, we have that $\left(R_{\ell}, \mathbf{a}_{\ell}\right) \neq\left(R_{\ell^{\prime}}, \mathbf{a}_{\ell^{\prime}}\right)$. A $\tau$-structure $\mathbb{T}$ is a $\tau$-forest if it has no circuits. If, additionally, it is connected then it is a $\tau$-tree. Usually, $\tau$-trees are defined using the notion of incidence multigraph (see for example [34]). It is easy to verify that the definition given here is equivalent.

\subsection{Forest of walks}

Given a $\tau$-structure $\mathbb{H}$, we proceed to define a new $\tau$-structure $\mathbb{T}_{\mathbb{H}}$. The universe $T_{\mathbb{H}}$ of $\mathbb{T}_{\mathbb{H}}$ consists of all the walks $w$ in $\mathbb{H}$. For a $k$-ary $R \in \tau$, we define $R\left(\mathbb{T}_{\mathbb{H}}\right)$ as follows: for all $\mathbf{a}=\left(a_{1}, \ldots, a_{k}\right) \in R(\mathbb{H})$, for all $1 \leq i \leq k$, and for all walks $w$ ending in $a_{i}$, we include in $R\left(\mathbb{T}_{\mathbb{H}}\right)$ the tuple $\left(w_{1}, \ldots, w_{i-1}, w, w_{i+1}, \ldots, w_{k}\right)$, where $w_{j}, j \neq i$, is the walk obtained from $w$ by extending it with $i,(R, \mathbf{a}), j, a_{j}$.

We note that $\mathbb{T}_{\mathbb{H}}$ does not have circuits and has exactly $|H|$ connected components, i.e., $|H| \tau$-trees. It is easy to check that for every substructure $\mathbb{I}$ of $\mathbb{H}$, the $\tau$-structure $\mathbb{T}_{\mathbb{I}}$ is a substructure of $\mathbb{T}_{\mathbb{H}}$.

- Remark 4. If $\mathbb{H}$ is connected and we consider a slight modification of this previous definition, where the walks are asked to be non-backtracking (i.e., for every $1 \leq \ell<n$, we have that either $i_{\ell} \neq j_{\ell+1}$, or $j_{\ell} \neq i_{\ell+1}$, or $\left.\left(R_{\ell}, \mathbf{x}_{\ell}\right) \neq\left(R_{\ell+1}, \mathbf{x}_{\ell+1}\right)\right)$, then we obtain that each connected component of the resulting $\tau$-structure corresponds to the universal covering tree of $\mathbb{H}[31,33]$ (in particular, they are all the same up to isomorphism).

Note that, by construction, the map $\rho_{\mathbb{H}}: T_{\mathbb{H}} \rightarrow H$ that sends every walk $w$ in $T_{\mathbb{H}}$ to its ending point, that from now on we refer as the label map, defines a homomorphism from $\mathbb{T}_{\mathbb{H}}$ to $\mathbb{H}$. Furthermore,

- Lemma 5. Assume that $\mathbb{H}$ is $J$-non-foldable for some $J \subseteq H$ and let $U$ be a cofinite subset of $T_{\mathbb{H}}$ containing $\rho_{\mathbb{H}}^{-1}(J)$. Then, every homomorphism in $\operatorname{Hom}\left(\mathbb{T}_{\mathbb{H}}, \mathbb{H}\right)$ that agrees with $\rho_{\mathbb{H}}$ in $U$ is identical to $\rho_{\mathbb{H}}$.

Proof. Given $n \geq 0$, let $W_{n}$ be the set of walks of length at least $n$ in $\mathbb{H}$ (notice that $W_{n} \subseteq W_{n-1}$ and $\left.W_{0}=T_{\mathbb{H}}\right)$. We shall show that any $\rho^{\prime} \in \operatorname{Hom}\left(\mathbb{T}_{\mathbb{H}}, \mathbb{H}\right)$ that agrees with $\rho_{\mathbb{H}}$ in $W_{n} \cup \rho_{\mathbb{H}}^{-1}(J)$ for arbitrary $n$ also agrees with $\rho_{\mathbb{H}}$ in $W_{n-1}$. Let $w$ be any walk of length $n-1$ and let $a$ be its ending point. We first show that $\rho^{\prime}(w)$ dominates $a$ in $\mathbb{H}$. Indeed, let $R \in \tau$ and let $\mathbf{a}=\left(a_{1}, \ldots, a_{k}\right) \in R(\mathbb{H})$, where $a$ appears, say, in the $i$ th coordinate. By construction, $R\left(\mathbb{T}_{\mathbb{H}}\right)$ contains the tuple $\mathbf{w}=\left(w_{1}, \ldots, w_{i-1}, w, w_{i+1}, \ldots, w_{k}\right)$, where for every $j \neq i, w_{j}$ is obtained by concatenating $i,(R, \mathbf{x}), j, a_{j}$ at the end of $w$. Since $w_{j}$ has length $n$ for every $j \neq i$, it follows by assumption that $\rho^{\prime}\left(w_{j}\right)=a_{j}$. That is, $\rho^{\prime}(\mathbf{w})$ (which must be a tuple in $R(\mathbb{H})$ ) is obtained by replacing, in $\mathbf{a}, a_{i}$ by $\rho^{\prime}(w)$. 
Hence, we have shown that $\rho^{\prime}(w)$ dominates $a$ in $\mathbb{H}$. Since $\mathbb{H}$ is $J$-non-foldable it follows that either $\rho^{\prime}(w)=a$ (and, hence, $\left.\rho^{\prime}(w)=\rho_{\mathbb{H}}(w)\right)$ or $a \in J$ (and, hence, $\rho^{\prime}(w)=\rho_{\mathbb{H}}(w)$ since $\left.w \in \rho_{\mathbb{H}}^{-1}(J)\right)$. To conclude the proof it is only necessary to observe that, since $U$ is a cofinite set containing $\rho_{\mathbb{H}}^{-1}(J)$, it follows that any homomorphism that agrees with $\rho_{\mathbb{H}}$ in $U$, agrees as well in $W_{n} \cup \rho_{\mathbb{H}}^{-1}(J)$ for sufficiently large $n$.

\subsection{Graphs of homomorphisms}

Let $\mathbb{G}$ and $\mathbb{H}$ be $\tau$-structures and suppose that $\mathbb{H}$ is finite. We define two different kinds of graphs with vertex set $\operatorname{Hom}(\mathbb{G}, \mathbb{H})$. The first notion has been heavily studied, from an algorithmic perspective, in the context of the so-called CSP reconfiguration problem (see [26] and the references therein) and, in the special cases when $\mathbb{G}$ and $\mathbb{H}$ are graphs also from an structural point of view $[12]$. We define $C(\mathbb{G}, \mathbb{H})$ as the (reflexive) graph with vertex set $\operatorname{Hom}(\mathbb{G}, \mathbb{H})$ such that for every $\phi, \psi \in \operatorname{Hom}(\mathbb{G}, \mathbb{H}), \phi$ and $\psi$ are adjacent if and only if $\phi$ and $\psi$ differ in at most one value, i.e., there exists at most one $x \in G$ such that $\phi(x) \neq \psi(x)$. More generally, for any $n \geq 1$ we can define $C_{n}(\mathbb{G}, \mathbb{H})$ on $\operatorname{Hom}(\mathbb{G}, \mathbb{H})$ by declaring $\phi$ and $\psi$ adjacent if they differ in at most $n$ values (in particular, $C(\mathbb{G}, \mathbb{H})=C_{1}(\mathbb{G}, \mathbb{H})$ ).

A second notion of graph of homomorphisms appears in [34] and uses the notion of links. The 1-link $\mathbb{L}$ (with signature $\tau$ ) is the $\tau$-structure with universe $\{0,1\}$, where $R(\mathbb{L})=\{0,1\}^{k}$ for every $k$-ary $R \in \tau$. Define a $($ di)graph $L(\mathbb{G}, \mathbb{H})$ with vertex set $\operatorname{Hom}(\mathbb{G}, \mathbb{H})$ as follows: set $\phi \rightarrow \psi$-i.e., a directed edge starting from $\phi$ and ending in $\psi$ - if for any $k$-ary $R \in \tau$ and any $\left(x_{1}, \ldots, x_{k}\right) \in R(\mathbb{G})$, we have that $\left(\gamma_{1}\left(x_{1}\right), \ldots, \gamma_{k}\left(x_{k}\right)\right) \in R(\mathbb{H})$ whenever $\gamma_{1}, \ldots, \gamma_{k} \in\{\phi, \psi\}$. Alternatively, one can say that there $\phi$ and $\psi$ are joined by a directed edge if there exists a homomorphism from $\mathbb{L}$ to $\mathbb{H}^{\mathbb{G}}$, the $\mathbb{H}$ th power of $\mathbb{G}$ (see [34, Section 5.2]), mapping 0 to $\phi$ and 1 to $\psi$. Notice that the symmetry in the definition of 1 -link implies that $L(\mathbb{G}, \mathbb{H})$ is, in fact, an undirected graph.

Clearly, $C_{n}(\mathbb{G}, \mathbb{H})$ is a subgraph of $C_{n+1}(\mathbb{G}, \mathbb{H})$. In contrast, $C_{n}(\mathbb{G}, \mathbb{H})$ and $L(\mathbb{G}, \mathbb{H})$ are not included in one another in general.

Note that there is a one-to-one correspondence between the elements in $\operatorname{Hom}(\mathbb{L} \times \mathbb{G}, \mathbb{H})$ and the edges of $L(\mathbb{G}, \mathbb{H})$. More generally, for $\ell \geq 1$ we define the $\ell$-link $\mathbb{L}_{\ell}$ (with signature $\tau$ ) as the $\tau$-structure with universe $\{0,1, \ldots, \ell\}$, where $R\left(\mathbb{L}_{\ell}\right)=\cup_{i=0}^{\ell-1}\{i, i+1\}^{k}$, for every $k$-ary $R \in \tau$. In other words, the $\ell$-link is a sequence of 1 -links with their endpoints identified. Then the following result is immediate:

Lemma 6. For every map $\phi:\{0,1, \ldots, \ell\} \times G \rightarrow H$ and every $1 \leq i \leq \ell$, let $\phi(i): G \rightarrow H$ be the map defined by $\phi(i)(x) \mapsto \phi(i, x)$ for $x \in G$. Then, $\phi \in \operatorname{Hom}\left(\mathbb{L}_{\ell} \times \mathbb{G}, \mathbb{H}\right)$ if and only if $\phi(0), \ldots, \phi(\ell)$ is a walk in $L(\mathbb{G}, \mathbb{H})$.

\subsection{Mixing properties}

Given $\tau$-structure $\mathbb{G}$ and $\mathbb{H}$, it is useful to study properties in $\operatorname{Hom}(\mathbb{G}, \mathbb{H})$ that allow us to glue together partially defined homomorphisms. This kind of properties are usually referred in the literature as mixing properties (e.g., see [8, 10]).

A natural mixing property is irreducibility. We say that $\operatorname{Hom}(\mathbb{G}, \mathbb{H})$ is $(V, W)$-irreducible for $V, W \subseteq G$, if for every $\phi, \psi \in \operatorname{Hom}(\mathbb{G}, \mathbb{H})$, there exists a map $\gamma \in \operatorname{Hom}(\mathbb{G}, \mathbb{H})$ that agrees with $\phi$ on $V$ and agrees with $\psi$ on $W$.

Given $g \geq 0$, we say that $\operatorname{Hom}(\mathbb{G}, \mathbb{H})$ is strongly irreducible with gap $g$ if for every $V, W$ such that $\operatorname{dist}(V, W) \geq g$ and for all $\phi, \psi \in \operatorname{Hom}(\mathbb{G}, \mathbb{H})$, there exists $\gamma \in \operatorname{Hom}(\mathbb{G}, \mathbb{H})$ that agrees with $\phi$ on $V$ and agrees with $\psi$ on $W$. We say that $\operatorname{Hom}(\mathbb{G}, \mathbb{H})$ is strongly irreducible if it is strongly irreducible with gap $g$ for some $g$. 
A strengthening of strong irreducibility is the following property, introduced in [7]. Given $g \geq 0$, we say that $\operatorname{Hom}(\mathbb{G}, \mathbb{H})$ is topologically strong spatial mixing (TSSM) with gap $g$ if for every $V, W, S \subseteq G$ such that $\operatorname{dist}(V, W) \geq g$ and for all $\phi, \psi \in \operatorname{Hom}(\mathbb{G}, \mathbb{H})$ that agree on $S$, there exists $\gamma \in \operatorname{Hom}(\mathbb{G}, \mathbb{H})$ that agrees with $\phi$ on $V \cup S$ and agrees with $\psi$ on $S \cup W$. We say that $\operatorname{Hom}(\mathbb{G}, \mathbb{H})$ is topologically strong spatial mixing if it is TSSM with gap $g$ for some $g$.

Clearly, $\operatorname{Hom}(\mathbb{G}, \mathbb{H})$ is TSSM only if $\operatorname{Hom}(\mathbb{G}, \mathbb{H})$ is strongly irreducible but not viceversa (see $[7,8]$ for some counterexamples).

An antithesis of having good mixing properties is the existence of configurations which are frozen. We say that $\phi \in \operatorname{Hom}(\mathbb{G}, \mathbb{H})$ is a frozen configuration if for any cofinite set $U \subseteq G$, the only homomorphism $\psi \in \operatorname{Hom}(\mathbb{G}, \mathbb{H})$ such that $\left.\psi\right|_{U}=\left.\phi\right|_{U}$ is $\psi=\phi$ itself.

\section{Dismantlability, Connectivity, and Irreducibility}

In this section we present our main theorem, which characterizes in several ways a special class of relational structures. This theorem generalizes some of the equivalences characterizing dismantlable graphs that appear in [12, Theorem 4.1] - which were developed only for the case of graphs - to arbitrary relational structures.

\subsection{The case of graphs}

The following theorem is a rephrasing of the equivalences that appear in [12, Theorem 4.1] which are relevant to us. We will use this as a prototypical example of the kind of results that we are aiming for, where we split the properties in 3 main categories (A) dismantlability, (B) connectedness, and (C) mixing.

Theorem 7 ([12, Theorem 4.1]). Let $\mathbb{H}$ be a graph. The following are equivalent:

(A) $\mathbb{H}$ is dismantlable;

(B) $C(\mathbb{G}, \mathbb{H})$ is connected for every locally finite graph $\mathbb{G}$;

(C) there exists $g \geq 0$ such that $\operatorname{Hom}(\mathbb{G}, \mathbb{H})$ is strongly irreducible with gap g for every graph $\mathbb{G}$.

Lemma 8. A graph $\mathbb{H}$ is dismantlable if and only if $\mathbb{H}^{2}$ dismantles to a substructure of its diagonal.

Proof. This follows from our own results. In Theorem 10, we prove that, for a finite $\tau$ structure $\mathbb{H}$, we have that $\mathbb{H}^{2}$ dismantles to a substructure of its diagonal if and only if there exists $g \geq 0$ such that $\operatorname{Hom}(\mathbb{G}, \mathbb{H})$ is strongly irreducible with gap $g$ for all $\tau$-structures $\mathbb{G}$. In particular, this applies if $\tau=\{E\}$, the usual binary relation of adjacency in graphs. Therefore, by Theorem 7 , these two properties are also equivalent to $\mathbb{H}$ being dismantlable, and we conclude.

In other words, thanks to Lemma 8, at least in the realm of graphs, we can freely replace "dismantlable" by "the square dismantles to a substructure of its diagonal", which will be the relevant class of general relational structures in this work.

- Remark 9. It is important to notice that the equivalence between "dismantlable" and "the square dismantles to a substructure of its diagonal" is not true for general relational structures. For example, given $\tau=\{R\}$ for $R$ a binary relation symbol, we can take $\mathbb{H}$ such that $H=\{0,1\}$ and $R(\mathbb{H})=\{(0,1)\}$. Then, $\mathbb{H}$ is not dismantlable, but $\mathbb{H}^{2}$ dismantles to its diagonal. 


\subsection{Main Theorem}

The following theorem shows that different dismantling, connectedness, and mixing notions are equivalent. It can be seen as a generalization of Theorem 7 to relational structures.

- Theorem 10. Let $\mathbb{H}$ be a finite $\tau$-structure with universe $H$. Then the following are equivalent:

(A1s) $\mathbb{H}$ dismantles to a structure $\mathbb{I}$ such that $\mathbb{I}^{2}$ dismantles to its diagonal;

(A2s) $\mathbb{H}^{2}$ dismantles to a substructure of its diagonal;

(B1s) $C(\mathbb{G}, \mathbb{H})$ is connected for every locally finite $\tau$-structure $\mathbb{G}$;

(B2s) there exists some $n \geq 1$ such that $C_{n}(\mathbb{G}, \mathbb{H})$ is connected for every finite $\tau$-structure

(B3s) $C\left(\mathbb{L} \times \mathbb{H}^{2}, \mathbb{H}\right)$ is connected;

(B4s) $L(\mathbb{G}, \mathbb{H})$ is connected for every finite $\tau$-structure $\mathbb{G}$;

(B5s) the projections $\pi_{1}$ and $\pi_{2}$ are connected in $L\left(\mathbb{H}^{2}, \mathbb{H}\right)$;

(C1s) there exists $g \geq 0$, such that $\operatorname{Hom}(\mathbb{G}, \mathbb{H})$ is strongly irreducible with parameter $g$ for every $\tau$-structure $\mathbb{G}$; and

(C2s) there exists $g \geq 0$, such that $\operatorname{Hom}\left(\mathbb{T}_{\mathbb{H}^{2}}, \mathbb{H}\right)$ is $(\{x\}, W)$-mixing with parameter $g$, for all $x \in T_{\mathbb{H}^{2}}$ and $W \subseteq T_{\mathbb{H}^{2}}$.

The proof of Theorem 10 can be found in the full version. Indeed, we prove a more general version of it which will allow us to derive the applications contained in the rest of the paper. Although due to space restrictions we cannot state the theorem in its more general form we believe is interesting in its own. In particular, it is motivated by the fact that, sometimes, it is natural - particularly in the context of statistical physics - to work by forcing a certain subset of particles to take each of them a particular spin and work with the remaining ones. For example, this is a common scenario when the particles in the boundary of a given set in a lattice are fixed to take particular spins and we want to study the distribution of spins in the interior of the set, conditioned on such boundary configuration. These ideas inspired the most general version, which can be regarded as the study of boundary long range actions, i.e., long range action phenomena where some boundary configuration is fixed, very similar to the concept of boundary phase transition in relation to phase transitions (e.g., see [38]).

\section{First application: Gibbs measures and mixing conditions}

\subsection{Basic definitions}

Given a finite $\tau$-structure $\mathbb{H}$ with universe $H$, a weight function for $\mathbb{H}$ is a map $\lambda: H \rightarrow \mathbb{R}^{+}$.

Let $\mathbb{G}$ be a locally finite $\tau$-structure. If $V \subseteq G$ is a finite set and $\phi \in \operatorname{Hom}(\mathbb{G}, \mathbb{H})$, we define $\mathbb{P}_{V, \phi}$ to be the probability measure on $\operatorname{Hom}(\mathbb{G}, \mathbb{H})$ given by

$$
\mathbb{P}_{V, \phi}(\{\psi\}):= \begin{cases}Z_{V, \phi}(\lambda)^{-1} \prod_{x \in V} \lambda(\psi(x)) & \text { if }\left.\left.\psi\right|_{V} \phi\right|_{G \backslash V} \in \operatorname{Hom}(\mathbb{G}, \mathbb{H}), \\ 0 & \text { otherwise }\end{cases}
$$

for $\psi \in \operatorname{Hom}(\mathbb{G}, \mathbb{H})$, where $\left.\left.\psi\right|_{V} \phi\right|_{G \backslash V}$ is the map that coincides with $\psi$ in $V$ and with $\phi$ in $G \backslash V$, and $Z_{V, \phi}(\lambda)$ is a normalization constant - the partition function - defined as

$$
Z_{V, \phi}(\lambda):=\sum_{\substack{\left.\left.\psi \in \operatorname{Hom}(\mathbb{G}, \mathbb{H}) \\ \psi\right|_{V} \phi\right|_{G \backslash V} \in \operatorname{Hom}(\mathbb{G}, \mathbb{H})}} \prod_{x \in V} \lambda(\psi(x))
$$


We will call the collection of probability measures $\left\{\mathbb{P}_{V, \phi}\right\}$, the Gibbs $(\mathbb{G}, \mathbb{H}, \lambda)$-specification. The boundary of a set $V \subseteq G$, denoted by $\partial V$, is defined as the set of elements in $G$ at distance exactly 1 from $V$. Notice that $\mathbb{P}_{V, \phi}$ depends exclusively on $\left.\phi\right|_{\partial V}$. Now, consider events of the form

$$
A(\phi, V)=\left\{\psi \in \operatorname{Hom}(\mathbb{G}, \mathbb{H}):\left.\psi\right|_{V}=\left.\phi\right|_{V}\right\} .
$$

Next, consider the $\sigma$-algebra $\mathcal{F}$ generated by all events of the form $A(\phi, V)$ for $V$ finite, and define $\mathcal{M}(\mathbb{G}, \mathbb{H})$ to be the set of probability measures on $(\operatorname{Hom}(\mathbb{G}, \mathbb{H}), \mathcal{F})$.

A measure $\mu \in \mathcal{M}(\mathbb{G}, \mathbb{H})$ is a Gibbs measure for the Gibbs $(\mathbb{G}, \mathbb{H}, \lambda)$-specification if for any finite $V \subseteq G$ and for all $\phi_{1} \in \operatorname{Hom}(\mathbb{G}, \mathbb{H})$,

$$
\mu\left(A\left(\phi_{1}, V\right) \mid A\left(\phi_{2}, G \backslash V\right)\right)=\mathbb{P}_{V, \phi_{2}}\left(\left\{\phi_{1}\right\}\right) \text { for } \mu \text {-a.e. } \phi_{2} \in \operatorname{Hom}(\mathbb{G}, \mathbb{H}) \text {. }
$$

In other words, the probability distribution of a random $\phi_{1}$ inside a finite $V$ conditioned on its values outside $V$ to coincide with those of $\phi_{2}$, depends only on the values of $\left.\phi_{1}\right|_{V}$ and on the boundary, $\left.\phi_{2}\right|_{\partial V}$. Furthermore, the conditional distribution is the same as for $\mathbb{P}_{V, \phi_{2}}$ (see also [12, Definition 2.1]).

If $\operatorname{Hom}(\mathbb{G}, \mathbb{H}) \neq \emptyset$, then there always exists at least one Gibbs measure [25, Chapter 4]. A fundamental question in statistical physics is whether there exists a unique Gibbs measure or multiple for a given Gibbs $(\mathbb{G}, \mathbb{H}, \lambda)$-specification.

\subsection{Non-uniqueness and spatial mixing properties}

In [12], it is shown that if $\mathbb{H}$ is a graph and it is dismantlable (or equivalently, by Lemma 8 , its square dismantles to a subgraph of its diagonal), then, for any locally finite graph $\mathbb{G}$, there exists some $\lambda$ such that there is a unique Gibbs measure [12, Theorem 7.2]. Conversely, in [12] it is also proven that if $\mathbb{H}$ is a non-dismantlable graph, then there exists $\mathbb{G}$ such that for any $\lambda$ there exists multiple Gibbs measures [12, Theorem 8.2].

Here, following a similar path, we show that when extending this question to arbitrary relational structures, the first implication does not remain true in general, but the second still holds. More exactly,

- Proposition 11. There exists a finite $\tau$-structure $\mathbb{H}$ such that $\mathbb{H}^{2}$ dismantles to a substructure of its diagonal and a locally finite $\tau$-structure $\mathbb{G}$ such that for any $\lambda$ there exists multiple Gibbs measures for the Gibbs $(\mathbb{G}, \mathbb{H}, \lambda)$-specification. Moreover, $\mathbb{H}$ can be chosen so that $\mathbb{H}^{2}$ dismantles to its full diagonal $\Delta\left(\mathbb{H}^{2}\right)$.

- Proposition 12. Let $\mathbb{H}$ be a finite $\tau$-structure. If $\mathbb{H}^{2}$ does not dismantle to a substructure of $\Delta\left(\mathbb{H}^{2}\right)$, then there exists a locally finite $\tau$-structure $\mathbb{G}$ such that for any $\lambda$ there exists multiple Gibbs measures for the Gibbs $(\mathbb{G}, \mathbb{H}, \lambda)$-specification.

In what follows we introduce some spatial mixing properties related to our results.

- Definition 13. Given $J \subseteq H$, we say that a Gibbs $(\mathbb{G}, \mathbb{H}, \lambda)$-specification satisfies spatial $J$-mixing (J-SM) if there exists constants $C, \alpha>0$ such that for all $\phi_{1}, \phi_{2} \in \operatorname{Hom}(\mathbb{G}, \mathbb{H})$, for all finite $V \subseteq G$, and for all $x \in V$ and $a \in H$,

$$
\left|\mathbb{P}_{V, \phi_{1}}(\{\psi(x)=a\})-\mathbb{P}_{V, \phi_{2}}(\{\psi(x)=a\})\right| \leq C \cdot \exp \left(-\alpha \cdot \operatorname{dist}\left(x, D_{V}^{J}\left(\phi_{1}, \phi_{2}\right)\right)\right),
$$

where

$$
D_{V}^{J}\left(\phi_{1}, \phi_{2}\right)=\left\{x \in \partial V:\left(\phi_{1}(x), \phi_{2}(x)\right) \in H^{2} \backslash \Delta\left(J^{2}\right)\right\}
$$

and $\{\psi(x)=a\}$ refers to the event that a random $\psi$ takes the value a at $x$. 
The definition of $J$-SM unifies and interpolates two well-known properties. If $J=\emptyset$, then $D_{V}^{\emptyset}\left(\phi_{1}, \phi_{2}\right)=\partial V$ and Eq. (1) corresponds to the definition of weak spatial mixing (WSM), i.e., $\emptyset$-SM. On the other hand, if $J=H$, then $D_{V}^{H}\left(\phi_{1}, \phi_{2}\right)=\left\{x \in \partial V: \phi_{1}(x) \neq\right.$ $\left.\phi_{2}(x)\right\}$ and Eq. (1) corresponds to the definition of strong spatial mixing (SSM), i.e., $H$-SM.

In general, spatial mixing properties are forms of correlation decay that have been of interest because of their many applications. On the one hand, WSM is related with uniqueness of Gibbs measures and the absence of phase transitions [21]. On the other hand, SSM is a strengthening of WSM and it is related to the absence of boundary phase transitions [38], and has connections with the existence of FPTAS for \#P-hard counting problems [3, 43], mixing time of Glauber dynamics [21], and efficient approximation algorithms for thermodynamic quantities $[24,7]$.

In [8], there were explored sufficient and necessary conditions for a graph $\mathbb{H}$ to have, for any locally finite graph $\mathbb{G}$, the existence of a weight function $\lambda$ such that the Gibbs $(\mathbb{G}, \mathbb{H}, \lambda)$ specification satisfies WSM and SSM. In particular, it was proven that dismantlability was equivalent to the existence of Gibbs $(\mathbb{G}, \mathbb{H}, \lambda)$-specifications satisfying WSM for all locally finite graph $\mathbb{G}$, and therefore uniqueness, since WSM implies it. In addition, it was observed that a direct consequence is that a necessary condition for SSM to hold is that $\mathbb{H}$ is dismantlable, because SSM implies WSM. However, it was also shown that it is not a sufficient condition. Here, we strengthen this necessary condition and extend it to the realm of relational structures.

- Proposition 14. If $\mathbb{H}^{2}$ does not dismantle to a substructure of $\Delta\left(\mathbb{H}^{2}\right)$ whose universe contains $\Delta\left(J^{2}\right)$, then there exists a locally finite $\tau$-structure $\mathbb{G}$ such that the Gibbs $(\mathbb{G}, \mathbb{H}, \lambda)$ specification does not satisfy $J-S M$ for any $\lambda$.

Two direct corollaries of this fact are the following.

- Corollary 15. If $\mathbb{H}^{2}$ does not dismantle to some substructure of the diagonal $\Delta\left(\mathbb{H}^{2}\right)$, then there exists a locally finite $\tau$-structure $\mathbb{G}$ such that the Gibbs $(\mathbb{G}, \mathbb{H}, \lambda)$-specification does not satisfy WSM for any $\lambda$.

- Corollary 16. If $\mathbb{H}^{2}$ does not dismantle to the full diagonal $\Delta\left(\mathbb{H}^{2}\right)$, then there exists a locally finite $\tau$-structure $\mathbb{G}$ such that the Gibbs $(\mathbb{G}, \mathbb{H}, \lambda)$-specification does not satisfy SSM for any $\lambda$.

\section{Second application: finite duality revisited}

Throughout this section all relational structures are assumed to be finite. We say a $\tau$-structure $\mathbb{H}$ is a core if every homomorphism from $\mathbb{H}$ to $\mathbb{H}$ is one-to-one. An obstruction to $\mathbb{H}$ is a $\tau$-structure $\mathbb{G}$ that admits no homomorphism to $\mathbb{H}$; the obstruction $\mathbb{G}$ is critical if every proper substructure (i.e., any substructure different from $\mathbb{G}$ itself) admits a homomorphism to $\mathbb{H}$. A relational structure $\mathbb{H}$ is said to have finite duality if it has only finitely many critical obstructions.

We say that a $\tau$-structure $\mathbb{H}$ contains all constants if for every $a \in H$ there exists $R_{a} \in \tau$ such that $R_{a}(\mathbb{H})=\{a\}$. Note that every such $\tau$-structure is a core. It is well know that relational structures with constants allow us to specify the desired image of a given element. More formally, let $\mathbb{G}$ be any $\tau$-structure, $x \in G$, and $a \in H$. It is immediate that the $\tau$-structure $\mathbb{G}_{a}$ obtained from $\mathbb{G}$ by adding $a$ to $R_{a}(\mathbb{G})$, satisfies the following property: For every $\phi: G \rightarrow H$,

$$
\phi \in \operatorname{Hom}\left(\mathbb{G}_{a}, \mathbb{H}\right) \Leftrightarrow \phi \in \operatorname{Hom}(\mathbb{G}, \mathbb{H}) \text { and } \phi(x)=a .
$$


We shall say that $\mathbb{G}_{a}$ is obtained by coloring $x$ to $a$ in $\mathbb{G}$.

The main result in [34] states that a core relational structure $\mathbb{H}$ has finite duality if and only if $\mathbb{H}^{2}$ dismantles to its diagonal. It is not difficult ot see that this result follows from our work. In addition, in can be shown that, when $\mathbb{H}$ contains all constants, having finite duality is equivalent to having finitely many critical $\tau$-tree obstructions, which was not previously known.

Theorem 17. Let $\mathbb{H}$ be a finite $\tau$-structure which is a core. Then, the following are equivalent:

(A1c) $\mathbb{H}^{2}$ dismantles to its diagonal;

(D1c) $\mathbb{H}$ has finitely many critical obstructions.

Furthermore, if $\mathbb{H}$ contains all the constants then the following condition is also equivalent: (D2c) $\mathbb{H}$ has finitely many critical $\tau$-tree obstructions.

It has been shown in [23] that if a $\tau$-structure $\mathbb{H}$ has finite duality then there exists some finite set $\mathcal{F}$ of $\tau$-trees such that for every $\tau$-structure $\mathbb{I}$ not homomorphic to $\mathbb{H}$, there exists a $\tau$-tree in $\mathcal{F}$ that is homomorphic to $\mathbb{I}$ but not homomorphic to $\mathbb{H}$. We want to note that the equivalence between conditions $(D 2 c)$ and $(D 1 c)$ does not follow from this fact. Indeed, direction $(D 2 c) \Rightarrow(D 1 c)$ does not even hold when we do not require that the $\tau$-structure $\mathbb{H}$ is equipped with constants as witnessed by the case when $\mathbb{H}$ is the oriented 3-cycle. Note that, in this case, $\mathbb{H}$ satisfies $(D 2 c)$ since every $\tau$-tree is homomorphic to $\mathbb{H}$ and, hence, $\mathbb{H}$ has no critical $\tau$-tree obstructions at all. However, since any oriented cycle whose length is not a multiple of 3 is a critical obstruction of $\mathbb{H}$, it follows that $\mathbb{H}$ does not satisfy $(D 1 c)$.

\section{References}

1 Dimitris Achlioptas, Paul Beame, and Michael Molloy. Exponential bounds for DPLL below the satisfiability threshold. In Proceedings of the Fifteenth Annual ACM-SIAM Symposium on Discrete Algorithms, SODA 2004, New Orleans, Louisiana, USA, January 11-14, 2004, pages 139-140, 2004.

2 Jung-Chao Ban and Chih-Hung Chang. Tree-shifts: Irreducibility, mixing, and the chaos of tree-shifts. Trans. Amer. Math. Soc., 369(12):8389-8407, 2017.

3 Antar Bandyopadhyay and David Gamarnik. Counting without sampling: Asymptotics of the log-partition function for certain statistical physics models. Random Structures \& Algorithms, 33(4):452-479, 2008.

4 Manuel Bodirsky. The Complexity of Constraint Satisfaction Problems (Invited Talk). In 32nd International Symposium on Theoretical Aspects of Computer Science, STACS 2015, March 4-7, 2015, Garching, Germany, pages 2-9, 2015.

5 Christian Borgs, Jennifer Chayes, László Lovász, Vera T Sós, and Katalin Vesztergombi. Counting graph homomorphisms. In Topics in discrete mathematics, pages 315-371. Springer, 2006.

6 Mike Boyle, Ronnie Pavlov, and Michael Schraudner. Multidimensional sofic shifts without separation and their factors. Trans. Amer. Math. Soc., 362(9):4617-4653, 2010.

7 Raimundo Briceño. The topological strong spatial mixing property and new conditions for pressure approximation. Ergodic Theory Dynam. Systems, 38(5):1658-1696, 2018.

8 Raimundo Briceño and Ronnie Pavlov. Strong spatial mixing in homomorphism spaces. SIAM J. Discrete Math., 31(3):2110-2137, 2017.

9 Raimundo Briceño, Andrei Bulatov, Víctor Dalmau, and Benoit Larose. Long range actions, connectedness, and dismantlability in relational structures. CoRR, abs/1901.04398, 2019 arXiv: 1901.04398. 
10 Raimundo Briceño, Kevin McGoff, and Ronnie Pavlov. Factoring onto $\mathbb{Z}^{d}$ subshifts with the finite extension property. Proc. Amer. Math. Soc., 146(12):5129-5140, 2018.

11 Graham R. Brightwell and Peter Winkler. Graph homomorphisms and phase transitions. J. Combin. Theory Ser. B, 77(2):221-262, 1999.

12 Graham R. Brightwell and Peter Winkler. Gibbs measures and dismantlable graphs. $J$. Combin. Theory Ser. B, 78(1):141-166, 2000.

13 Graham R. Brightwell and Peter Winkler. Graph homomorphisms and long range action. DIMACS Series in Discrete Mathematics and Theoretical Computer Science, 63:29-48, 2004.

14 Andrei A. Bulatov. A Dichotomy Theorem for Nonuniform CSPs. In 58th IEEE Annual Symposium on Foundations of Computer Science, FOCS 2017, Berkeley, CA, USA, October 15-17, 2017, pages 319-330, 2017.

15 Andrei A. Bulatov, Víctor Dalmau, Martin Grohe, and Dániel Marx. Enumerating homomorphisms. J. Comput. Syst. Sci., 78(2):638-650, 2012.

16 Andrei A. Bulatov, Andrei A. Krokhin, and Benoit Larose. Dualities for Constraint Satisfaction Problems. In Complexity of Constraints - An Overview of Current Research Themes [Result of a Dagstuhl Seminar], pages 93-124, 2008.

17 Tullio Ceccherini-Silberstein and Michel Coornaert. On the density of periodic configurations in strongly irreducible subshifts. Nonlinearity, 25(7):2119, 2012.

18 Víctor Dalmau, Andrei A. Krokhin, and Benoit Larose. First-Order Definable Retraction Problems for Posets and Reflexive Graph. In 19th IEEE Symposium on Logic in Computer Science (LICS 2004), 14-17 July 2004, Turku, Finland, Proceedings, pages 232-241, 2004.

19 Rina Dechter. Constraint processing. Morgan Kaufmann, 2003.

20 Martin Dyer and Catherine Greenhill. The complexity of counting graph homomorphisms. Random Structures \& Algorithms, 17(3-4):260-289, 2000.

21 Martin Dyer, Alistair Sinclair, Eric Vigoda, and Dror Weitz. Mixing in time and space for lattice spin systems: A combinatorial view. Random Structures \&6 Algorithms, 24(4):461-479, 2004.

22 Tomás Feder and Moshe Y. Vardi. The computational structure of monotone monadic SNP and constraint satisfaction: A study through Datalog and group theory. SIAM Journal on Computing, 28(1):57-104, 1998.

23 Jan Foniok, Jaroslav Nesetril, and Claude Tardif. Generalised dualities and maximal finite antichains in the homomorphism order of relational structures. Eur. J. Comb., 29(4):881-899, 2008.

24 David Gamarnik and Dmitriy Katz. Sequential cavity method for computing free energy and surface pressure. Journal of Statistical Physics, 137(2):205, 2009.

25 Hans-Otto Georgii. Gibbs Measures and Phase Transitions, volume 9 of De Gruyter Studies in Mathematics. Berlin, 2 edition, 2011.

26 Tatsuhiko Hatanaka, Takehiro Ito, and Xiao Zhou. Complexity of Reconfiguration Problems for Constraint Satisfaction. CoRR, abs/1812.10629, 2018.

27 Pavol Hell and Jaroslav Nešetřil. Graphs and homomorphisms, volume 28 of Oxford Lecture Series in Mathematics and its Applications. Oxford University Press, Oxford, 2004.

28 Takehiro Ito, Erik D. Demaine, Nicholas J. A. Harvey, Christos H. Papadimitriou, Martha Sideri, Ryuhei Uehara, and Yushi Uno. On the complexity of reconfiguration problems. Theor. Comput. Sci., 412(12-14):1054-1065, 2011.

29 David Kelly and Ivan Rival. Crowns, Fences, and Dismantlable Lattices. Canadian Journal of Mathematics, 26(5):1257-1271, 1974.

30 P. Kolaitis and M. Vardi. Conjunctive-Query Containment and Constraint Satisfaction. Journal of Computer and System Sciences, 61:302-332, 2000.

31 Marcin Kozik. Weak consistency notions for all the CSPs of bounded width. In 31st Annual ACM/IEEE Symposium on Logic in Computer Science, LICS '16, pages 633-641, 2016. 
32 Florent Krzakała, Andrea Montanari, Federico Ricci-Tersenghi, Guilhem Semerjian, and Lenka Zdeborová. Gibbs states and the set of solutions of random constraint satisfaction problems. PNAS, 104(25):10318-10323, 2007.

33 Gábor Kun, Ryan O’Donnell, Suguru Tamaki, Yuichi Yoshida, and Yuan Zhou. Linear programming, width-1 CSPs, and robust satisfaction. In Innovations in Theoretical Computer Science, ICT '12, pages 484-495, 2012.

34 Benoit Larose, Cynthia Loten, and Claude Tardif. A characterisation of first-order constraint satisfaction problems. Log. Methods Comput. Sci., 3(4):4:6, 22, 2007.

35 Douglas Lind and Brian Marcus. An introduction to symbolic dynamics and coding. Cambridge university press, 1995.

36 Douglas Lind and Klaus Schmidt. Symbolic and algebraic dynamical systems. In Handbook of dynamical systems, volume 1, pages 765-812. Elsevier, 2002.

37 Brian Marcus and Joachim Rosenthal. Codes, systems, and graphical models, volume 123. Springer Science \& Business Media, 2012.

38 Fabio Martinelli, Enzo Olivieri, and Roberto H Schonmann. For 2-D lattice spin systems weak mixing implies strong mixing. Communications in Mathematical Physics, 165(1):33-47, 1994

39 Marc Mézard and Andrea Montanari. Information, Physics, and Computation. Oxford University press, 2009.

40 Naomi Nishimura. Introduction to Reconfiguration. Algorithms, 11(4):52, 2018.

41 Richard Nowakowski and Peter Winkler. Vertex-to-vertex pursuit in a graph. Discrete Mathematics, 43(2-3):235-239, 1983.

42 Ronnie Pavlov and Michael Schraudner. Entropies realizable by block gluing $\mathbb{Z}^{d}$ shifts of finite type. Journal d'Analyse Mathématique, 126(1):113-174, 2015.

43 Dror Weitz. Counting Independent Sets Up to the Tree Threshold. In Proceedings of the Thirty-eighth Annual ACM Symposium on Theory of Computing, STOC '06, pages 140-149, New York, NY, USA, 2006. ACM.

44 Dmitriy Zhuk. A Proof of CSP Dichotomy Conjecture. In 58th IEEE Annual Symposium on Foundations of Computer Science, FOCS 2017, Berkeley, CA, USA, October 15-17, 2017, pages 331-342, 2017. 